\title{
Human Papillomaviruses Infection In Oral Squamous Cell Carcinoma And Oral Pre-cancer Lesions In Taiwan
}

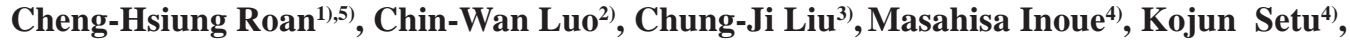 \\ Hitoshi Nagatsuka5) and Noriyuki Nagai ${ }^{5)}$
}

\author{
1) Department of Pathology and Gyn, Taipei Medical University Hospital, Taipei, Taiwan \\ 2) Department of Oral Surgery, Taipei Medical University Hospital, Taipei, Taiwan \\ 3) Department of Oral and Maxillofacial Surgery, Mackay Memorial Hospital, Taiwan \\ 4) Laboratories for Structure and Function Research, Faculty of Pharmaceutical Sciences Tokushima Bunri University, Tokushima, \\ Japan \\ 5) Department of Oral Pathology and Medicine, Graduate School of Medicine, Dentistry and Pharmaceutical sciences, Okayama \\ University, Okayama, Japan
}

\begin{abstract}
Human papilloma viruses (HPV) infection is a significant risk factor for uterine cervical carcinoma. Many previous studies have also demonstrated the presence of HPVs in oral epithelia tissue. However, the role of HPV infection in oral squamous cell carcinoma (OSCC) is still controversy. The present study is to determine the frequency and type of HPV in OSCCs and oral pre-cancer lesions. Methods: DNA samples were collected from 51 OSCCs, 46 oral pre-cancer lesions and 90 normal control specimens by cytobrushings. Nested polymerase chain reaction (PCR), and gene-chip are used to identify multiple HPV types in our samples. Results: The positive rates of overall HPV types $(14 / 46, \mathrm{P}=0.0216, \mathrm{OR}=2.844, \mathrm{CI}=1.186-6.816)$ and of lowrisk types (9/46, $\mathrm{P}=0.0096, \mathrm{OR}=5.529, \mathrm{CI}=1.597-19.14)$ are significantly higher in oral pre-cancer lesions than in control samples. The prevalence of high-risk type (11/51, $\mathrm{P}=0.0420, \mathrm{OR}=2.819, \mathrm{CI}=1.051-7.558)$ is significantly higher in OSCCs than in control but of overall types $(13 / 51, \mathrm{P}=0.1066$, OR=2.244, CI=0.92665.337 ) is not to reach the statistical significance.Conclusion: The high-risk HPV may play a role in OSCCs progression and the low-risk ones may associate with the oral pre-cancer lesions.
\end{abstract}

Keywords: human papillomavirus, genotype, cytobrush, gene-chip, mouth neoplasm.

\section{Introduction}

Squamous cell carcinoma is the most common malignant neoplasm of the oral mucosa, representing more than $90 \%$ of these malignant tumors. In Taiwan, oral cancer ranks as the seventh most prevalent cancer in both sexes and account for the fourth most common cancer in males ${ }^{1)}$. Known risk factors for oral squamous cell carcinoma (OSCC) are long-term tobacco, alcohol, and betel quit use; irradiation; viruses and chronic irritants. Numerous persons are exposed to these risk factors, only small proportions of these individuals have OSCCs developed. The findings suggest that other factors may contribute a role in oral carcinogenesis.

Human papillomavirus (HPV) are small, nonenveloped, icosahedral, epitheliotropic DNA viruses. To date more than 100 different HPV genotypes have been cloned and characterized ${ }^{2}$. The HPVs infections are associated with a large spectrum of epithelial lesions. Most of these are benign hyperplasia. A subgroup of HPVs, i.e., the high-risk HPVs included 16, 18, 31, $33,35,39,45,52$.are associated with lesions that have a propensity to undergo carcinogenesis ${ }^{3), 4)}$. These subgroups have been suggested to play an important role in the epithelial carcinomas of the uterine cervix. ${ }^{210}$ During cancer progression, the high-risk virus frequently becomes integrated into the host genome. After integration, two viral genes, E6 and E7, are consistently expressed. The E6 and E7 genes of the HPVs encode oncoproteins that target the p53 and retinoblastoma tumor suppressors result in loss of control over the cell cycle ${ }^{3)}$.

The oral cavity, like the cervical area, is covered by squamous epithelium with or without slight keratinization and continuously exposed to the external environment. Many previous studies have also demonstrated the presence of HPVs in oral epithelial tissue ${ }^{4)}$. However, the relationship of HPVs to OSCCs remain unclear because of the difficulties in interpreting studies that demonstrate various prevalence rates of HPVs. The purpose of this study, we investigate the prevalence and typing of HPVs infection from patients with different types of mucosal lesions and compared these with samples from a group of normal control subjects without oral mucosal disease. The hypothesis is that the specific genotypes of HPV may play a role in malignant transformation. We adopt the cytology brush method to collect the exfoliated cells, Nested polymerase chain reaction (PCR)-based gene-chip is used to determine multiple HPV types. This assay has been proved to be reliable for identify multiple HPVs types ${ }^{5), 10)}$.

\section{Materials and Methods}

The study procedures are first DNA extraction,than HPV detection by Nested PCR followed genotyping by gene chip.The study results were analysis by Fisfer's exact test.

Total 97 patients were included in this study. The 51 of them (48 men and 3 women; range, 33 - 71 years) were with OSCCs, and the 46 of them (45 men and 1 women; range, 20 - 74 years) were with oral pre-cancers lesion include leukoplakia, and verrucous hyperplasia. The diagnosis was based on histologic examination of H\&E stained tissue section, during the period from January 2002 to May 2004.

Cytological specimens were obtained from the lesions of each subject before the operation. The brush was held against the mucosa of the lesion and rotated for ten full turns. The brush kept 
International symposium of Maxillofacial \& Oral Regenerative Biology in Okayama 2005

\begin{tabular}{cc}
\hline Primer & Sequence \\
\hline MY11 & 5' GCM CAG GGW CAT AAY AAT GG \\
GP5+ & 5' TTT GTT ACT GTG GTA GAT AC \\
GP6+ & 5' GAA AAA TAA ACT GTA AAT CA \\
\hline
\end{tabular}

Table 1:Human Papillomavirus Sequence Primers Used for Polymerase Chain Reaction

into the HBSS solution in $4^{\circ} \mathrm{C}$ until further procedures. Of the 51 cases of OSCCs, there were 10 (19.61\%) tongue, 27 (52.94\%) buccal, 10 (19.61\%) gingiva, 2 (3.92\%) lip, and 2 (3.92\%) floor of mouths cancer. The histologic features of OSCCs were classified into 3 types (well-differentiated, moderately differentiated, poorly differentiated OSCCs). Of the 51 cases of OSCCs, there were 22 (43.13\%) well-differentiated, 25 (49.01\%) moderately differentiated, and 4 (7.84\%) poorly differentiated.

90 specimens of normal oral mucosa were obtained from 90 subjects (52 men and 38 women; range, 15 - 75 years). The DNA samples were collected by the same procedure at the appointment for extraction of impacted permanent lower third molars during the period from January 2002 to January 2004 used as control samples.

\section{Results}

Amplification of the human $\beta$-actin gene in all DNA samples indicated that sufficient DNA was present for the amplification of HPV sequences. To rule out the possibility of contamination and PCR artifacts, at least 3 negative control samples containing all reaction components except template DNA were included for each round of PCR amplification. No PCR product was detected in the first or second round of amplification of the negative control samples, indicating the absence of contamination.

We used a nested PCR-based gene-chip method to determine multiple HPV types in OSCCs, oral pre-cancer and control samples. HPV positive rates, the ORs and 95\% C.I. for OSCCs and oral pre-cancer patients compared to control subjects are given in table 2, 3 and 4. The HPV DNA detection rate was higher in oral pre-cancer lesion patients $(14 / 46,30.43 \%)$ than in control subjects (12/90, 13.33\%; $\mathrm{P}=0.0216)$. The HPV-positive rates were also higher in OSCCs patients (13/51, 25.49\%) than in control subjects but did not reach a clear statistical difference $(\mathrm{P}=0.106)$.
The positive rates of HPV DNA in OSCCs were not associated with the tumor location and histologic feature. In HPV-positive OSCCs patients, high-risk subgroup type was found more significantly frequently than control subjects $(\mathrm{P}=0.0420)$. The HPV low-risk subgroup positive-rates were significantly higher in oral pre-cancer patients than OSCCs $(\mathrm{P}=0.0227)$ and control subjects $(\mathrm{P}=0.0096)$. However, there was no significant correlation in high-risk subgroup HPV positive rate between oral pre-cancer group and control subjects.

\section{Discussion}

Previous studies have provided some evidence of an association between HPV and the development of OSCCs. ${ }^{4}$ The discrepancy in the results for HPV detection rate from different studies may be due to the difference of the detection methods and the use of the tissue samples. According to the present review by Miller et al, ${ }^{4}$, assays consider to be of low sensitivity included immunoperoxidase, immunofluorescence, and in situ hybridization. Assays consider to be of moderate sensitivity included Southern blot, dot blot hybridization. Assays consider to be of high sensitivity used the polymerase chain reaction (PCR). The detection rates are highest with the PCR assays. So we use the nested PCR-based gene-chip assay to detect the HPV. The review by Miller suggest that HPV DNA detection rates about 46.5\% in OSCCs, $22.2 \%$ in benign lesions and $10.0 \%$ in normal subjects ${ }^{4}$. Compare to our date, the positive rate in normal subjects (13.3\%) and oral pre-malignant (30.43\%) lesions is higher, but lower in OSCCs group (25.49\%). Factors contributing to the difference include the study sample size, and regionalism. Our study adopts the fresh cytobrushing specimens and with a nested PCR-based gene-chip method to determine multiple HPV types. This assay could be considered easily to perform and noninvasive, with high sensitivity and the results also to be reliable. The result of this study shows that the HPV-positive rate in OSCCs tissue samples is higher than in control subjects but not reach statistical significance. When focus on the prevalence of high-risk subgroup HPV infection, it is significantly higher in OSCCs group. On the other hand, the overall HPV prevalence is significantly high in oral pre-malignant samples. The major subgroup of HPV infected genotype in oral pre-malignant lesions is low-risk subgroup type. The positive rates are significantly higher than OSCCs and control subjects. These findings support the hypothesis that the specific genotypes of HPV may play a role in malignant transformation.

Table 2:HPV positive rate, Odds Ratios and 95\% C.I. for OSCCs

\begin{tabular}{llclcl}
\hline HPV type & OSCC $(\mathrm{n}=51)$ & control $(\mathrm{n}=90)$ & Odds Ratio & 95\% C.I. & P value \\
\hline All & $13(25.49 \%)$ & $12(13.33 \%)$ & 2.224 & $0.9266-5.337$ & 0.1066 \\
High-risk & $11(21.57 \%)$ & $8(8.89 \%)$ & 2.819 & $1.051-7.558$ & $0.0420^{*}$ \\
Low-risk & $2(3.92 \%)$ & $4(4.44 \%)$ & 0.8776 & $0.1550-4.968$ & 1.000 \\
\hline
\end{tabular}

HPV: human papillomavirus C.I: confidence interval *: statistical significance

Table 3:HPV positive rate, Odds Ratios and 95\% C.I. for oral pre-cancer lesions

\begin{tabular}{|c|c|c|c|c|c|}
\hline HPV type & $\begin{array}{l}\text { oral pre-cancer } \\
(\mathrm{n}=46)\end{array}$ & control $(n=90)$ & Odds Ratio & 95\% C.I. & $\mathrm{P}$ value \\
\hline All & $14(30.43 \%)$ & 12 (13.33\%) & 2.844 & $1.186-6.816$ & $0.0216 *$ \\
\hline High-risk & $5(10.87 \%)$ & $8(8.89 \%)$ & 1.25 & $0.3845-4.063$ & 0.7618 \\
\hline Low-risk & $9(19.57 \%)$ & $4(4.44 \%)$ & 5.529 & $1.597-19.14$ & $0.0096 *$ \\
\hline
\end{tabular}


HPV positive rate, Odds Ratios and 95\% C.I. for OSCCs and oral pre-cancer lesions

\begin{tabular}{llllll}
\hline HPV type & OSCCs $(\mathrm{n}=51)$ & $\begin{array}{l}\text { Oral pre-cancer } \\
(\mathrm{n}=46)\end{array}$ & $\begin{array}{l}\text { Odds } \\
\text { Ratio }\end{array}$ & 95\% C.I. & P value \\
\hline All & $13(25.49 \%)$ & $14(30.43 \%)$ & 0.7820 & $0.3212-1.903$ & 0.6534 \\
High-risk & $11(21.57 \%)$ & $5(10.87 \%)$ & 2.255 & $0.7186-7.077$ & 0.1808 \\
Low-risk & $2(3.92 \%)$ & $9(19.57 \%)$ & 0.1678 & $0.03419-0.82369$ & $0.0227^{*}$ \\
\hline
\end{tabular}

C.I: confidence interval $\quad *$ : statistical significance

High-risk genotypes HPV infecting the oral epithelium have the potential to propagate new malignancies. On the other hand, the low-risk genotypes infecting the oral epithelium may retard the progression of malignant transformation of oral pre-cancer lesion under the same environmental risk factors. the infecting HPV types may also an important prognostic factor. Schwartz have suggested that HPV DNA is a strong, independent positive prognostic marker in oral cancer, even though the infected tumors were more advanced ${ }^{9)}$.

\section{Conclusion}

The high-risk HPV may play a role in OSCCs progression and the low-risk ones may associate with the pre-cancer lesions. There are important that further studies of the molecular evidence about the inference of specific type HPV infection in oral epithelial tumor progression and the prognostic value.

\section{References}

1. Cancer Registry Annual Report in Taiwan Area, 1999, Taipei, Taiwan: Department of Health, The Executive Yuan; 2003

2. Cooper K. The role of human papillomaviruses in cervical cancer. S Afr Med J 85: 841-2, 1995

3. Alani RM, MungerK. Human papillomaviruses and associated malignancies. J Clin Oncol 16: 330-7, 1998

4. Miller CS, Johnstone BM. Human papillomavius as a risk factor for oral squamous cell carcinoma: a meta-analysis, 1982-1997. Oral Surg Oral Med Oral Pathol Oral Radiol Endod 91: 622-35, 2001
5. Chang JYF, Lin MC, Chiang CP. High-risk human papillomaviruses may have an important role in non-oral habits-associated oral squamous cell carcinomas in Taiwan. Am J Clin Pathol 120: 909-16, 2003

6. Premoli-De-Percoco G, Ramifez JL, Galindo I. Correlatin between HPV types associated with oral squamous cell carcinoma and cervicovaginal cytology: An in situ hybridization study. Oral Surg Oral Med Oral Pathol Oral Radiol Endod 86: 77-81, 1998

7. Miller CS, White DK. Human papillomavirus expression in oral mucosa, premalignant conditions, and squamous cell carcinoma: a retrospective review of the literature. Oral Surg Oral Med Oral Pathol Oral Radiol Endod 82: 57-68, 1996

8. Chang KW, Chang CS, Lai KS, Chou MJ, Choo KB. High prevalence of human papillomavirus infection and possible association with betel quit chewing and smoking in oral epidermoid carcinomas in Taiwan. J Med Virol 28: 57-61,1989

9. Schwartz SR, Yueh B, Mcdougall JK, Daling JR, Schwartz SM. Human papillomavirus infection and survival in oral squamous cell cancer:a population \based study. Otolaryngol Head Neck Surg 125;1-9, 2001

10. Huang HJ, Huang SL, Lin CY et al. Human papillomavirus genotyping by a PCR-based genochip method in cervical carcinoma treated with neoadjuvant chemotherapy plus radical surgery, Int J Cancer. In press. 